\title{
Diagnostic Value of Ascitic Tumor Markers for Gastric Cancer-associated Malignant Ascites
}

\author{
Yaewon Yang ${ }^{1}$, Hong Jun Kim², Se-il Go ${ }^{2}$, Woo Kyun Bae ${ }^{3,4}$, Eun-Kee Song ${ }^{5,6}$, Seonggyu Byeon ${ }^{1}$, Hee Kyung Kim', Yusook Jeong ${ }^{1}$, \\ Jihyun Kwon ${ }^{1,7}$, Ki Hyeong Lee ${ }^{1,7}$, Hee Bok Chae ${ }^{1,7}$, Seung-Myoung Son ${ }^{8,9}$, Dae Hoon Kim ${ }^{10}$, Hyo Yung Yun ${ }^{10,11}$, Hye Sook Han ${ }^{1,7}$ \\ Department of Internal Medicine, Chungbuk National University Hospital ${ }^{1}$, Cheongiu, Department of Internal Medicine, Gyeongsang National \\ University School of Medicine ${ }^{2}$, Jinju, Department of Internal Medicine, Chonnam National University Hwasun Hospital ${ }^{3}$, Hwasun, Department \\ of Internal Medicine, Chonnam National University Medical School ${ }^{4}$, Gwangju, Department of Internal Medicine, Chonbuk National University \\ Medical School, Research Institute of Clinical Medicine of Chonbuk National University, Biomedical Research Institute of Chonbuk National \\ University ${ }^{6}$, Jeonju, Department of Internal Medicine, Chungbuk National University College of Medicine ${ }^{7}$, Department of Pathology, Chungbuk \\ National University Hospital ${ }^{8}$, Department of Pathology, Chungbuk National University College of Medicine ${ }^{9}$, Department of Surgery, Chungbuk \\ National University Hospital ${ }^{10}$, Department of Surgery, Chungbuk National University College of Medicine ${ }^{11}$, Cheongju, Korea
}

Background/Aims: Peritoneal carcinomatosis with malignant ascites is common in patients with advanced gastric cancer (GC). The detection of tumor cells is the gold standard for the diagnosis of malignant ascites; however, it often requires complementary tests because of its low sensitivity. Herein, we measured the levels of tumor markers in the malignant ascites of GC patients (GC-ascites) and benign ascites of liver cirrhosis patients (LC-ascites) to elucidate the diagnostic value of tumor markers in GC-ascites.

Materials and Methods: The levels of CEA, cancer antigen 72-4 (CA 72-4), CA 19-9, and CA 125 were measured in 138 GC-ascites and $64 \mathrm{LC}$-ascites samples obtained from the National Biobank of Korea. We performed receiver operating characteristic curve analysis to determine the optimal cutoff value for each tumor marker.

Results: CEA, CA 72-4, and CA 19-9 levels were significantly higher in GC-ascites than in LC-ascites. There was no difference in tumor marker levels between GC-ascites samples irrespective of cytology. CEA, CA 72-4, and CA 19-9 had sensitivities of 85.5\%, $79.0 \%$, and $61.6 \%$, respectively, and specificities of $96.8 \%, 100.0 \%$, and $89.1 \%$, respectively, for distinguishing GC-ascites samples from LC-ascites samples. The diagnostic accuracy was improved by combining two or more tumor markers. The combination of CEA and CA 72-4 showed the highest sensitivity (86.2\%) and specificity (100\%).

Conclusions: Measurement of tumor markers, such as CEA, CA 72-4, and CA 19-9, in ascites samples could help diagnose GC-ascites, and combining two or more tumor markers could further increase the diagnostic yield, even in cytology-negative patients. (Korean J Helicobacter Up Gastrointest Res 2022;22:38-49)

Key Words: Ascites; Biomakers, tumor; Diagnosis; Stomach neoplasms

\section{INTRODUCTION}

Gastric cancer (GC) is the fifth most common cancer worldwide and the fourth leading cause of cancer-related mortality, based on the GLOBOCAN 2020 data estimates. Peritoneal carcinomatosis occurs in more than one-third of patients with advanced GC with metastatic disease, and the prognosis is generally poor. ${ }^{2}$ Early and accurate diagnosis of peritoneal carcinomatosis may increase the

Received: November 18, 2021 Revised: December 12, 2021 Accepted: December 26, 2021 Corresponding author: Hye Sook Han

Department of Internal Medicine, Chungbuk National University Hospital, Chungbuk National University College of Medicine, 1 Chungdae-ro, Seowon-gu, Cheongju 28644, Korea

Tel: +82-43-269-6306, Fax: +82-43-273-3252, E-mail: sook3529@hanmail.net

*This research was supported by the Basic Science Research Program through the National Research Foundation of Korea (NRF), funded by the Ministry of Education, Science and Technology (2017R1A5A2015541 and 2019R1A2C1007401). chances of appropriate treatment and improve the prognosis of patients with advanced GC. Malignant ascites is common in GC patients with peritoneal carcinomatosis. Therefore, differential diagnosis of the ascites is important to confirm the nature of the ascites as benign or malignant. Cytologic confirmation is the gold standard for the diagnosis of malignant ascites, although the sensitivity of the test is low. Although broad efforts have been made to improve the diagnostic accuracy and sensitivity of tests for malignant ascites with peritoneal carcinomatosis using tumor markers, the appropriate markers and their role in GC patients with ascites are still inconclusive.

A tumor marker is a molecule that indicates the likely presence of cancer or provides information about the likely future behavior of a cancer. Therefore, tumor markers are potentially useful in screening, diagnosis, de-

Copyright $\odot 2022$ Korean College of Helicobacter and Upper Gastrointestinal Research

(a) The Korean Journal of Helicobacter and Upper Gastrointestinal Research is an Open-Access Journal. All articles are distributed under the terms of the Creative Commons Attribution Non-Commercial License (http:// creativecommons.org/licenses/by-nc/4.0) which permits unrestricted non-commercial use, distribution, and reproduction in any medium, provided the original work is properly cited. 
termining prognosis, predicting therapeutic efficacy, surveillance following surgical removal, and monitoring treatment response in cancer patients. ${ }^{3}$ Serum tumor markers are most widely investigated in GC patients for predicting recurrence or prognosis, including CEA, CA 19-9, CA 125, and CA 72-4. ${ }^{4-6}$ Tumor markers in ascites samples have also been investigated in several studies, but their diagnostic role for malignant ascites in GC patients is still obscure. In most previous studies, the sample size was small, and the studied populations were not confined to GC patients. ${ }^{7-10}$

To investigate the diagnostic role of tumor markers in ascites samples, we measured and compared the levels of various tumor markers in GC-associated malignant ascites (GC-ascites) samples and liver cirrhosis (LC)-associated benign ascites (LC-ascites) samples. We also assessed the tumor markers of diagnostic significance and established the optimal cutoff value with the maximum sensitivity and specificity to distinguish GC-associated malignant ascites from benign ascites.

\section{MATERIALS AND METHODS}

\section{Patients and ascites samples}

The 202 ascites samples (64 LC-ascites samples and 138 GC-ascites samples) originated from four different centers, were collected at the National Biobank of Korea between January 2012 and April 2018. The LC-ascites samples were obtained from patients diagnosed with LC on a clinical and laboratory basis by a hepatologist. All patients had no past medical history or radiological evidence of any malignancy, and the absence of malignant cells in the LC-ascites samples was confirmed by cytological analysis. The GC-ascites samples were obtained from patients with confirmed diagnosis of primary gastric or gastroesophageal junction adenocarcinoma without any evidence of double primary malignancies. The definition of GC-ascites in this study was ascites in GC patients with either positive cytology in the ascites sample or with combined typical clinical and radiological findings despite of negative cytology. As the aim of current study was to elucidate the role of ascites tumor markers for the diag- nosis of $\mathrm{GC}$-ascites if the cytologic examination results were negative, only patients with clear evidence of peritoneal carcinomatosis were enrolled in the analyses to minimize the possibility of the false positives. Radiologic parameters suggestive of peritoneal metastasis and malignant ascites were as follows: (1) enhancing thickening of the bowel walls, (2) increased density of the peritoneal fat, (3) the presence of peritoneal seeding nodules, and (4) hydronephrosis from ureteral obstruction. ${ }^{11}$ Clinical conditions that strongly suggestive of peritoneal carcinomatosis included that continuous increase of ascites needed repetitive therapeutic paracentesis, or the occurrence of bowel obstruction, urinary tract obstruction, or biliary obstruction as a complication of peritoneal metastasis. The samples were collected during diagnostic or therapeutic paracentesis procedures from LC and GC patients who presented with ascites. After collection, the ascitic samples were sent to a pathologist for cytological analysis. A separate set of ascitic samples were centrifuged at 3,200×g for 10 minutes to eliminate large cell particles and cell debris. Supernatant from each sample was aliquoted into microcentrifuge tubes and stored at $-80^{\circ} \mathrm{C}$ for further analyses, including the measurement of tumor markers. Clinicopathological data were obtained from a retrospective review of the medical records.

The study protocol was reviewed and approved by the Institutional Review Board of the Chungbuk National University Hospital, South Korea (IRB approval number: CBNUH 2017-05-014), and written informed consent was obtained from all study participants at the time of ascites sample collection.

\section{Tumor marker measurement in ascites samples}

Based on the review of previous studies of commonly used tumor markers in gastric cancer, we selected CEA, CA 19-9, CA 72-4, and CA 125 to investigate the diagnostic role of ascites tumor markers. ${ }^{4,12-15}$ We measured the level of these four tumor markers in the LC- and GC-ascites samples. The levels of CEA, CA 72-4, CA 19-9, and $\mathrm{CA} 125$ in the ascites samples were quantified using an Architect i4000SR chemiluminescent enzyme immunoassay (Abbott Laboratories, Chicago, IL, USA) in accord- 
ance with the manufacturer's instructions.

\section{Statistical analyses}

Mann-Whitney unpaired test were performed to assess differences in the levels of tumor markers between LC-ascites and GC-ascites samples. The unpaired $t$-test was used to compare mean values and Chi-squared test was used to compare the differences. To determine the optimal cutoff value to maximize the diagnostic value of each tumor marker, we performed receiver operating characteristic (ROC) curve analysis and calculated the area under the curve (AUC). Risk scores were assigned to all patients in accordance with a linear combination between the levels of the tumor markers, weighted in accordance with the regression coefficient. Furthermore, we conducted stepwise Cox regression and stratification analyses. The statistical analyses were performed using IBM SPSS Statistics software version 21.0 (IBM Corp., Armonk, NY, USA) and MedCalc software version 18.9.1 (MedCalc, Ostend, Belgium). All the $P$-values were two-sided, and
$P$-values below 0.05 were considered statistically significant.

\section{RESULTS}

\section{Study population}

The baseline characteristics of the study population are summarized in Table 1. Of the 202 ascites samples, 138 (68.3\%) were GC-ascites and 64 (31.7\%) were LC-ascites. There were no significant differences in age and sex between the two groups. The most common cause of LC was chronic hepatitis B virus infection (60.8\%), followed by alcohol abuse (26.6\%) and chronic hepatitis $\mathrm{C}$ virus infection (6.3\%). For primary GC, tubular adenocarcinoma (56.5\%) was the most common histology, followed by poorly cohesive carcinoma (23.9\%) and mucinous carcinoma (4.3\%). Ninety-six ascites samples in GC-ascites (69.6\%) were cytologically positive for adenocarcinoma.

Table 1. Patient Characteristics

\begin{tabular}{|c|c|c|c|}
\hline & \multicolumn{2}{|c|}{ No. of patients $(n=202)$} & \multirow{2}{*}{$P$-value } \\
\hline & LC-ascites $(n=64)$ & GC-ascites $(n=138)$ & \\
\hline Age (years) & $59.0 \pm 12.8$ & $64.0 \pm 13.3$ & 0.118 \\
\hline Sex & & & 0.119 \\
\hline Male & $50(78.1)$ & $93(67.4)$ & \\
\hline Female & $14(21.9)$ & $45(32.6)$ & \\
\hline \multicolumn{4}{|l|}{ Etiology of liver cirrhosis } \\
\hline Hepatitis B virus infection & $39(60.8)$ & & \\
\hline Hepatitis $\mathrm{C}$ virus infection & $4(6.3)$ & & \\
\hline Alcohol abuse & $17(26.6)$ & & \\
\hline Unknown & $4(6.3)$ & & \\
\hline Histological classification of primary tumor & & & - \\
\hline \multicolumn{4}{|l|}{ Tubular adenocarcinoma } \\
\hline Well-differentiated & & $4(2.9)$ & \\
\hline Moderately differentiated & & $38(27.5)$ & \\
\hline Poorly differentiated & & $36(26.1)$ & \\
\hline Mucinous carcinoma & & $6(4.3)$ & \\
\hline Poorly cohesive carcinoma & & $33(23.9)$ & \\
\hline Mixed adenocarcinoma & & $5(3.6)$ & \\
\hline Unknown & & $16(11.6)$ & \\
\hline
\end{tabular}

Values are presented as mean \pm standard deviation or number (\%).

LC, liver cirrhosis; GC, gastric cancer. 


\section{Levels of tumor markers in ascites samples}

The levels of CEA, CA 72-4, and CA 19-9 were significantly higher in GC-ascites samples than in LC-ascites samples $(P<0.001)$, whereas the levels of $\mathrm{CA} 125$ were similar between the two groups ( $P=0.811)$ (Table 2, Fig. 1). Therefore, subsequent ROC curve analyses to evaluate the diagnostic value of tumor markers in GC-ascites were

Table 2. Levels of CEA, CA 72-4, CA 19-9, and CA 125

\begin{tabular}{|c|c|c|c|c|c|c|}
\hline \multirow{4}{*}{ Tumor marker } & \multicolumn{6}{|c|}{ Levels of tumor markers } \\
\hline & \multirow{2}{*}{\multicolumn{2}{|c|}{ LC-ascites vs. GC-ascites }} & \multirow{2}{*}{\multicolumn{3}{|c|}{$\begin{array}{c}\text { GC-ascites } \\
\text { Positive cytology vs. negati }\end{array}$}} & \multirow{3}{*}{$P$-value } \\
\hline & & & & & & \\
\hline & LC-ascites $(n=64)$ & GC-ascites $(n=138)$ & & Positive cytology $(n=96)$ & Negative cytology $(n=42)$ & \\
\hline CEA (ng/mL) & $0.72 \pm 0.66$ & $617.8 \pm 1896.98$ & $<0.001$ & $611.03 \pm 2078.58$ & $633.21 \pm 1418.84$ & 0.447 \\
\hline CA 72-4 (U/mL) & $1.50 \pm 0.03$ & $367.25 \pm 2226.17$ & $<0.001$ & $476.09 \pm 2663.75$ & $118.46 \pm 166.36$ & 0.492 \\
\hline CA 19-9 (U/mL) & $7.13 \pm 11.20$ & $1,860.52 \pm 5,882.67$ & $<0.001$ & $2,154.75 \pm 6,825.11$ & $1,188.00 \pm 2,652.13$ & 0.880 \\
\hline CA-125 (U/mL) & $564.62 \pm 369.32$ & $666.90 \pm 720.83$ & 0.811 & $694.30 \pm 709.53$ & $204.27 \pm 750.98$ & 0.198 \\
\hline
\end{tabular}

Values are presented as mean \pm standard deviation.

CEA, carcinoembryonic antigen; CA 72-4, cancer antigen 72-4; CA 19-9, carbohydrate antigen 19-9; CA-125, cancer antigen 125; LC, liver cirrhosis; $\mathrm{GC}$, gastric cancer.
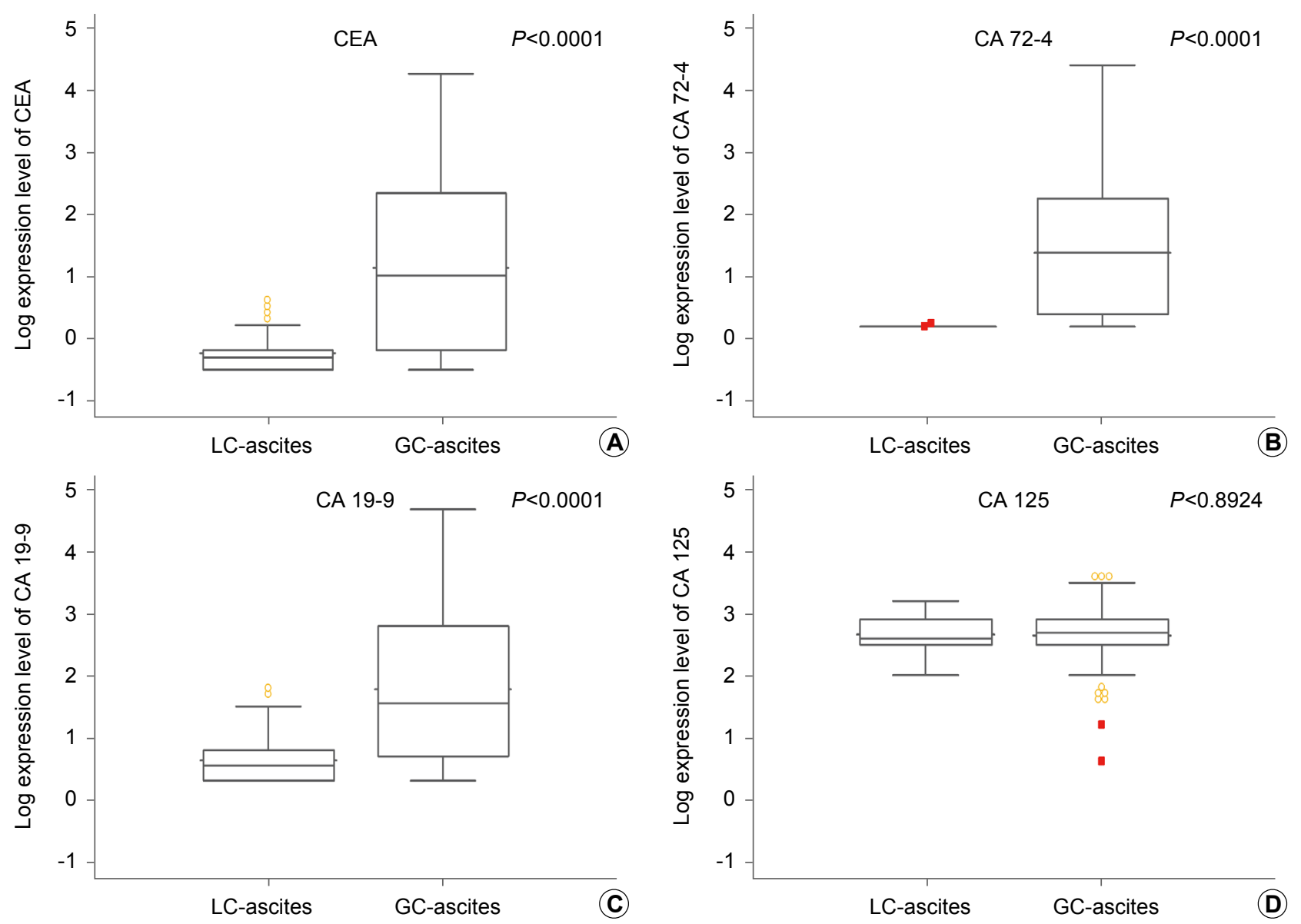

Fig. 1. Comparison of CEA (A), CA 72-4 (B), CA 19-9 (C), and CA 125 (D) levels in LC-associated ascites samples and GC-associated ascites samples. Statistical significance was determined by the Mann-Whitney test. CEA, carcinoembryonic antigen; CA 72-4, cancer antigen 72-4; LC, liver cirrhosis; GC, gastric cancer; CA 19-9, carbohydrate antigen 19-9; CA 125, cancer antigen 125. 
performed only for CEA, CA 72-4, and CA 19-9. The levels of tumor markers were slightly higher in GC-ascites samples with positive cytology than in samples with negative cytology, but this was not statistically significant (Table 2).

\section{Diagnostic value of the levels of tumor markers for GC-ascites}

We performed ROC curve analyses of CEA, CA 72-4, and CA 19-9 to determine their accuracy for diagnosing GC-ascites (Table 3, Fig. 2). CEA at a cutoff value of 2.3 $\mathrm{ng} / \mathrm{mL}$ had the highest sensitivity (85.5\%) and specificity (96.9\%), with an AUC of 0.952 (95\% CI, 0.912 0.977). CA $72-4$ at a cutoff value of $1.7 \mathrm{U} / \mathrm{mL}$ had $79.0 \%$ sensitivity and 100\% specificity (AUC, 0.892; 95\% CI, 0.840 0.924), and CA 19-9 at a cutoff of $10.2 \mathrm{U} / \mathrm{mL}$ had $61.6 \%$ sensitivity and 89.0\% specificity (AUC, 0.788; 95\% CI, 0.726 0.843) (Table 3, Fig. 2). These cutoff values are lower than the upper normal limits in serum samples of CEA, CA 72-4, and CA 19-9 $(5.0 \mathrm{ng} / \mathrm{mL}, 4.0 \mathrm{U} / \mathrm{mL}$, and $37 \mathrm{U} / \mathrm{mL}$, respectively).

Combining CEA and one or more other tumor markers improved the sensitivity and specificity for the diagnosis of GC-ascites (Table 3, Fig. 3). Combining CEA and CA 72-4 as the numerical sum of each value with a cutoff value of 5.1 had $86.2 \%$ sensitivity and $100.0 \%$ specificity (AUC, 0.966; 95\% CI, 0.930 0.986), and combining CEA and CA 19-9 with a cutoff value of 19.9 had $80.4 \%$ sensitivity and 93.8\% specificity (AUC, 0.939; 95\% CI, 0.897 0.968). The sum of all three tumor markers with a cutoff value of 21.4 had $85.5 \%$ sensitivity and $93.8 \%$ specificity,

Table 3. Diagnostic Value of Tumor Markers for the Diagnosis of Gastric Cancer-associated Malignant Ascites

\begin{tabular}{|c|c|c|c|c|c|c|c|}
\hline Tumor marker & Cutoff value & Sensitivity (\%) & Specificity (\%) & PPV (\%) & NPV (\%) & YI & AUC (95\% CI) \\
\hline \multicolumn{8}{|l|}{ Single tumor marker } \\
\hline CEA (ng/mL) & 2.3 & 85.5 & 96.9 & 98.4 & 75.6 & 0.8238 & $0.952(0.912 \sim 0.977)$ \\
\hline CA $72-4(\mathrm{U} / \mathrm{mL})$ & 1.7 & 79.0 & 100.0 & 100.0 & 68.8 & 0.7899 & $0.892(0.840 \sim 0.924)$ \\
\hline CA 19-9 (U/mL) & 10.2 & 61.6 & 89.0 & 92.3 & 51.8 & 0.5066 & $0.788(0.726 \sim 0.843)$ \\
\hline \multicolumn{8}{|c|}{ Combination of tumor markers } \\
\hline CEA+CA 72-4 & 5.1 & 86.2 & 100.0 & 100.0 & 77.1 & 0.8623 & $0.966(0.930 \sim 0.986)$ \\
\hline CEA+CA 19-9 & 19.9 & 80.4 & 93.8 & 96.5 & 69.0 & 0.7428 & $0.939(0.897 \sim 0.968)$ \\
\hline CEA+CA 72-4+CA 19-9 & 21.4 & 85.5 & 93.8 & 96.7 & 75.0 & 0.7926 & $0.950(0.910 \sim 0.976)$ \\
\hline
\end{tabular}

PPV, positive predictive value; NPV, negative predictive value; YI, Youden index; AUC, area under the curve; CI, confidence interval; CEA, carcinoembryonic antigen; CA 72-4, carbohydrate antigen 72-4; CA 19-9, carbohydrate antigen 19-9.

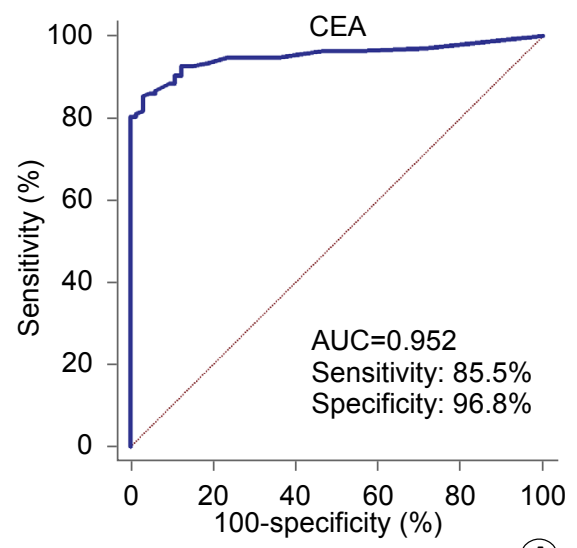

(A)
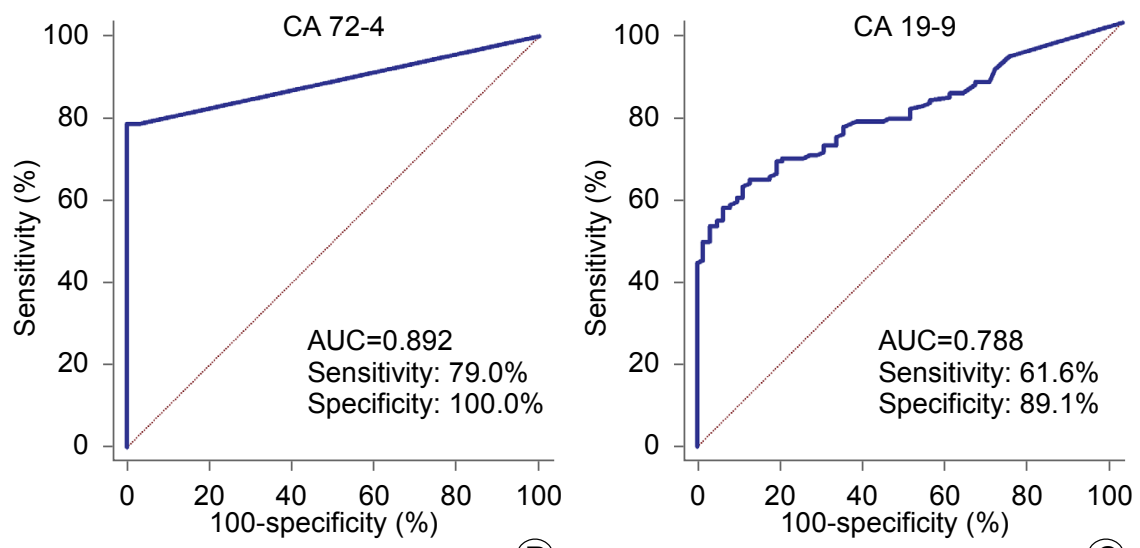

B
(C)

Fig. 2. Receiver operating characteristic curve analysis of single tumor markers for the diagnosis of gastric cancer-associated malignant ascites. AUC estimations for (A) CEA, (B) CA 72-4, and (C) CA 19-9. AUC, area under the curve; CEA, carcinoembryonic antigen; CA 72-4, cancer antigen 72-4; CA 19-9, carbohydrate antigen 19-9. 

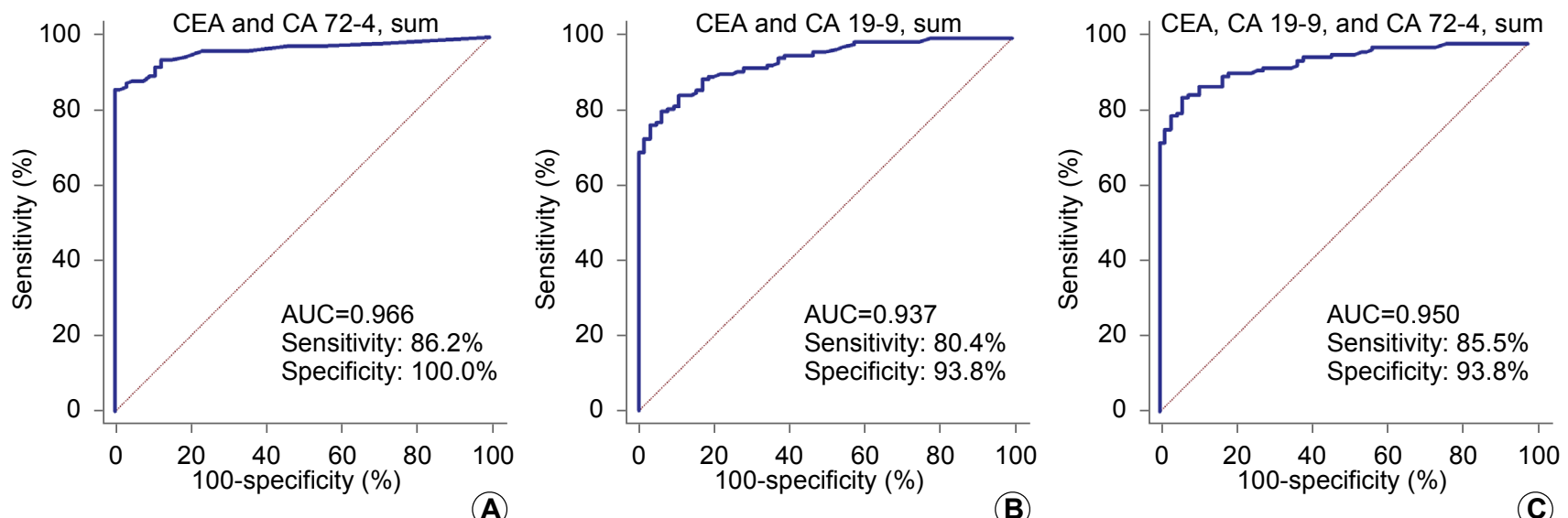

(B)

(C)

Fig. 3. Receiver operating characteristic curve analysis of the combination of tumor markers for the diagnosis of gastric cancer-associated malignant ascites as the numerical sum of each tumor marker. AUC estimations for (A) CEA plus CA 72-4, (B) CEA plus CA 19-9, and (C) CEA plus CA 72-4 plus CA 19-9. AUC, area under the curve; CEA, carcinoembryonic antigen; CA 72-4, cancer antigen 72-4; CA 19-9, carbohydrate antigen 19-9.

Table 4. Diagnostic Value of Combining Tumor Markers for the Diagnosis of Gastric Cancer-associated Malignant Ascites

\begin{tabular}{llcccccc}
\hline Tumor marker combinations & Diagnostic criteria & Sensitivity (\%) & Specificity (\%) & PPV (\%) & NPV (\%) & YI & AUC (95\% CI) \\
\hline CEA and CA 72-4 & $\begin{array}{l}\geq 1 \text { marker } \\
\text { above cutoff value }\end{array}$ & 87.7 & 96.9 & 98.4 & 78.5 & 0.8456 & $0.935(0.891 \sim 0.965)$ \\
CEA and CA 19-9 & $\begin{array}{c}\geq 1 \text { marker } \\
\text { above cutoff value }\end{array}$ & 90.6 & 85.9 & 93.3 & 80.9 & 0.7652 & $0.922(0.876 \sim 0.955)$ \\
CEA, CA 72-4, and CA 19-9 & $\begin{array}{l}\geq 2 \text { markers } \\
\text { above cutoff value }\end{array}$ & 81.2 & 100.0 & 100.0 & 71.2 & 0.8116 & $0.943(0.902 \sim 0.971)$ \\
\hline
\end{tabular}

PPV, positive predictive value; NPV, negative predictive value; YI, youden index; AUC, area under the curve; CI, confidence interval; CEA, carcinoembryonic antigen; CA 72-4, carbohydrate antigen 72-4; CA 19-9, carbohydrate antigen 19-9.

${ }^{a}$ The cutoff values of the ascites tumor markers in this study were determined to maximize the diagnostic sensitivity and specificity in distinguishing gastric cancer-associated malignant ascites from liver cirrhosis-associated benign ascites. Details on this analysis are presented in Table 3.

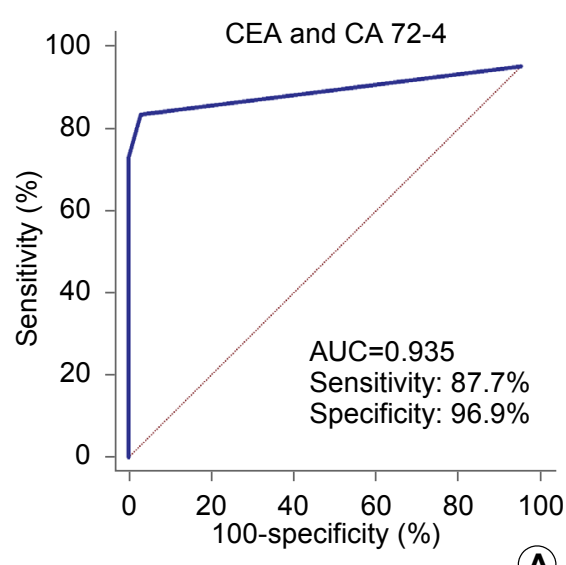

(A)

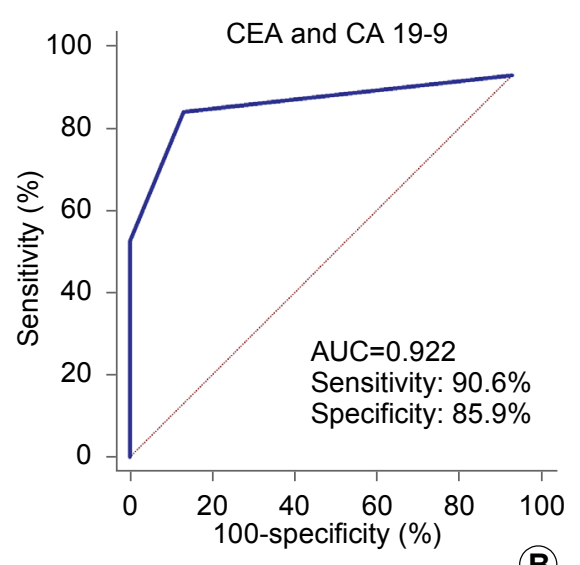

(B)

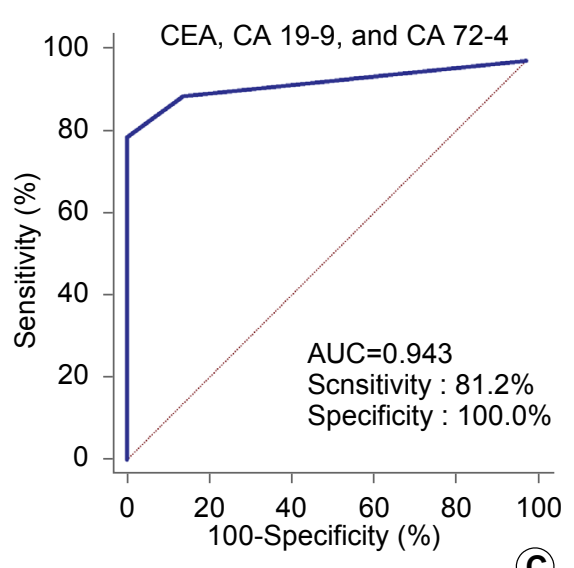

(C)

Fig. 4. Receiver operating characteristic curve analysis of the combination of tumor markers for the diagnosis of gastric cancer-associated malignant ascites using the number of tumor markers above the cutoff value. AUC estimations for (A) CEA and CA $72-4$ with $\geq 1$ tumor markers above the cutoff value, (B) CEA and CA 19-9 with $\geq 1$ tumor markers above the cutoff value, and (C) CEA, CA 72-4, and CA 19-9 with $\geq 2$ tumor markers above the cutoff. AUC, area under the curve; CEA, carcinoembryonic antigen; CA 72-4, cancer antigen 72-4; CA 19-9, carbohydrate antigen 19-9. 


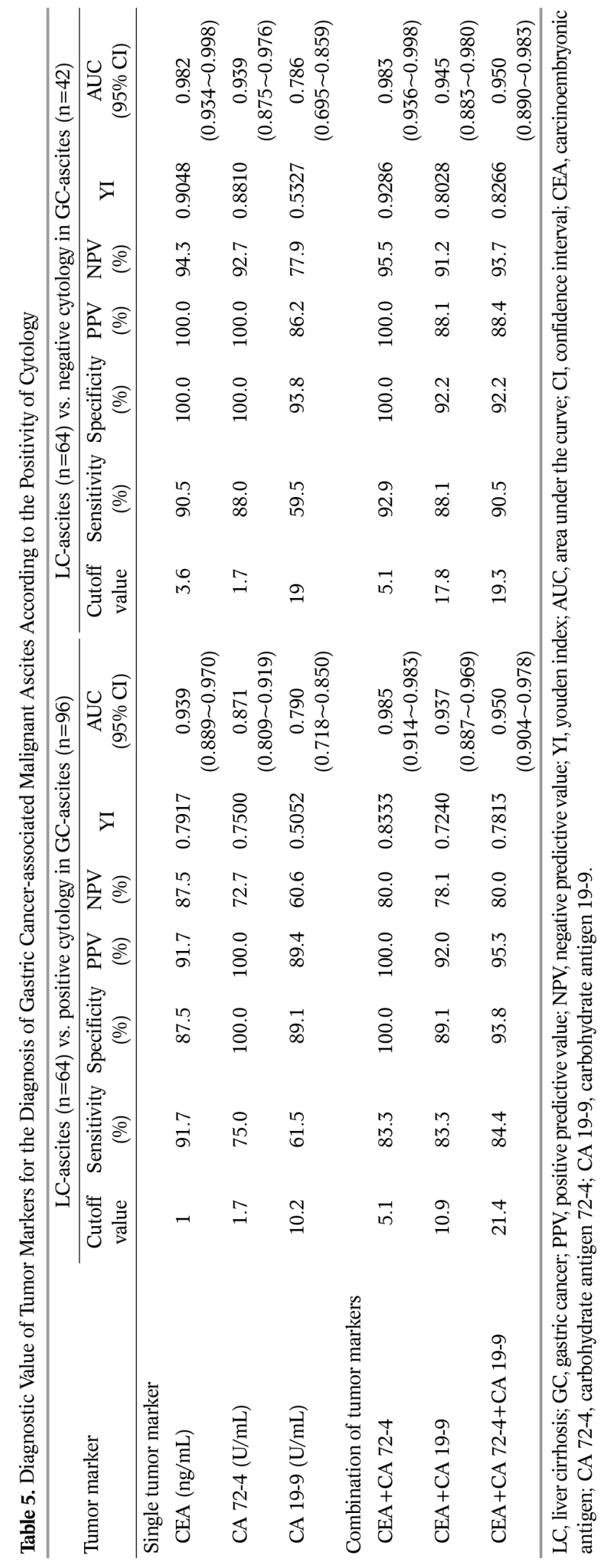

with an AUC of 0.950 (95\% CI, 0.910 0.976). We also analyzed the diagnostic value of combining CEA and other tumor markers not as the numerical sum but as the number of tumor markers above their corresponding cutoffs. When CEA, CA 72-4, or both were above their respective cutoff values, the AUC for the combination was 0.935 (95\% CI, 0.891 0.965), with a sensitivity of $87.7 \%$ and a specificity of $96.6 \%$. When CEA, CA 19-9, or both were above their respective cutoff values, the AUC was 0.922 (95\% CI, 0.876 0.955), with a sensitivity of $90.6 \%$ and a specificity of $85.9 \%$. When combining all three markers, when at least two markers were above their respective cutoff values, the AUC was 0.943 (95\% CI, 0.902

0.971), with a sensitivity of $81.2 \%$ and a specificity of 100\% (Table 4, Fig. 4). The overall results were similar to that of the combination method using the numerical sum and had better sensitivity and specificity than using a single ascites tumor marker. When using the numerical sum of the ascites tumor markers, the combination of CEA and CA 72-4 had the largest AUC (0.966). When using the number of tumor markers above the optimal cutoff value, the combination of all three tumor markers in ascites had the largest AUC (0.943; 95\% CI, 0.902 0.971).

We also performed the ROC curve analyses of tumor markers in both cytology positive and negative group, separately (Table 5). CEA had highest sensitivity and specificity for both groups for the diagnosis of GC-ascites, with AUC of 0.939 (95\% CI, 0.889 0.970) and 0.982 (0.934 0.998), respectively. CA $72-4$ and CA 19-9 also showed similar power for GC-ascites diagnosis in cytology positive and negative group. Combination of ascites tumor markers also showed similar results. Combination of CEA and CA 72-4 had highest diagnostic accuracy with AUC of 0.985 (95\% CI, 0.914 0.983) and 0.983 (95\% CI, 0.936 0.998) in cytology positive and negative group, respectively.

\section{DISCUSSION}

Here, we reported the role of ascites tumor markers in the diagnosis of GC-related malignant ascites. The levels of CEA, CA 72-4, and CA 19-9 were significantly higher in malignant GC-ascites than in benign LC-ascites. Tumor 
marker levels in GC-ascites were not different irrespective of the positivity of cytology. The sensitivity of the cytological analysis was $69.6 \%$, whereas CEA with a cutoff value of $2.3 \mathrm{ng} / \mathrm{mL}$ had $85.5 \%$ sensitivity and $96.9 \%$ specificity (AUC, 0.952; youden index [YI], 0.8223). The levels of CA 72-4 and CA 19-9 were also significantly higher in GC-ascites than in LC-ascites, but their low sensitivities limited their diagnostic roles as single biomarkers. When two or more tumor markers, including CEA, were combined, the accuracy for diagnosing GC-ascites was improved. The combination of CEA and CA 72-4 had $86.2 \%$ sensitivity and 100\% specificity (AUC, 0.966; YI, 0.862) for diagnosing GC-ascites. Ascites tumor markers showed similar diagnostic power for GC-ascites in both cytology positive and negative groups. Regarding the rather low sensitivity of cytological examination, combined analysis of ascites tumor markers could improve the diagnostic accuracy of GC-ascites.

The peritoneum is the most common metastatic site in advanced GC. An epidemiologic study in the Netherlands found peritoneal carcinomatosis in 35\% of GC patients with metastatic disease, and peritoneal carcinomatosis was the only metastatic site in $69.5 \%$ of these patients. GC patients with peritoneal carcinomatosis usually have poor prognosis, with a median overall survival of only 4.8 moths $(95 \% \mathrm{CI}, 3.5 \sim 5.7)$, which is shorter than that of all GC patients (7.9 months; 95\% CI, 7.2 9.1). ${ }^{2}$ Ascites is common in advanced GC patients with peritoneal carcinomatosis. Early diagnosis of peritoneal carcinomatosis and timely adequate treatment could improve the prognosis of these patients. Therefore, when ascites is detected, an accurate and prompt differential diagnosis should be made to determine whether the ascites is benign or malignant. Detection of tumor cells in ascites is the indisputable gold standard for the diagnosis of malignant ascites, although the sensitivity of the test is rather low. Explorative laparotomy to obtain peritoneal tissue may also be considered, but it is a time-consuming and relatively invasive procedure to perform as a routine test for diagnosing peritoneal carcinomatosis. Imaging procedures such as computed tomography or magnetic resonance imaging of the abdomen and pelvic cavity could assist in the diagnosis of peritoneal carcinomatosis, but their role in detecting early lesions with a small tumor burden is limited because of their low sensitivity.

Many studies have been conducted to improve the diagnostic accuracy for GC using tumor markers. Serum CEA, CA 19-9, CA 72-4, and CA-125 are the tumor markers that are most widely investigated and used in GC for diagnosis and prognosis prediction. ${ }^{4}$ Serum levels of CEA, CA 19-9, and CA 72-4 are significantly higher in GC patients than in healthy volunteers and in patients with benign gastric diseases and are considered to increase the accuracy for the diagnosis of GC. ${ }^{6}$ Serum CA 125 and CA 72-4 are useful markers in GC diagnosis, as well as in predicting prognosis and evaluating treatment efficacy in patients with GC peritoneal dissemination. ${ }^{5}$ Based on the efficacy of serum tumor markers, studies have also evaluated the diagnostic value of tumor markers in the peritoneal lavage fluid (PLF) after gastrectomy (Table 6). High CEA and CA 72-4 in the PLF are useful markers for the prediction of peritoneal recurrence or prognosis in GC patients who receive surgical resection, and elevated $\mathrm{CA}$ 19-9 in the PLF is significantly associated with advanced GC. 12,14 CEA levels or CEA and CA 125 levels in combination with peritoneal cytology in the PLF are helpful predictors of peritoneal dissemination in GC. ${ }^{15}$ CEA protein and mRNA levels in the PLF have high diagnostic accuracy and can be helpful to predict peritoneal recurrence after curative surgery in GC patients. ${ }^{16}$ Tumor markers in ascites samples have also been investigated in several studies, and efforts have been made to differentiate benign and malignant ascites using ascites tumor markers (Table 6). Adding other biomarkers also improves the diagnostic accuracy for malignant ascites. Combined analysis of exosomal miRNAs and tumor markers improves the diagnostic accuracy for GC-ascites compared to CEA alone. The combination of miR-181b-5p and CEA has $82.5 \%$ sensitivity and $96.7 \%$ specificity. ${ }^{17}$ However, none of these previous studies confirmed the diagnostic role of commonly used serum tumor markers in ascites samples for malignant ascites. Some studies suggested that the combination of serum tumor markers, cytology, or ascites heteroploid cells with ascites tumor markers could improve the diagnostic accuracy for malignant ascites, but those studies were not confined to GC. ${ }^{7-10}$ Therefore, our 
Table 6. Published Studies of Tumor Markers in the Peritoneal Lavage Fluid and Ascites of Gastric Cancer

\begin{tabular}{|c|c|c|c|c|c|c|c|c|}
\hline \multirow{2}{*}{ Samples } & \multirow{2}{*}{ Study } & \multicolumn{2}{|c|}{ No. of patients } & \multirow{2}{*}{ Tumor markers } & \multirow{2}{*}{ Cutoff } & \multirow{2}{*}{$\begin{array}{c}\text { Sensitivity } \\
(\%)\end{array}$} & \multirow{2}{*}{$\begin{array}{l}\text { Specificity } \\
(\%)\end{array}$} & \multirow{2}{*}{ Role of tumor markers } \\
\hline & & $\begin{array}{l}\text { Gastric } \\
\text { cancer }\end{array}$ & Control & & & & & \\
\hline \multirow[t]{19}{*}{ PLF } & \multirow[t]{3}{*}{$\begin{array}{l}\text { Yamamoto et } \\
\text { al. }^{21}(2004)\end{array}$} & \multirow[t]{3}{*}{229} & & CEA & $0.5 \mathrm{ng} / \mathrm{mL}$ & 75.8 & 90.8 & \multirow[t]{3}{*}{$\begin{array}{l}\text { Prediction of peritoneal } \\
\text { dissemination }\end{array}$} \\
\hline & & & & CA 125 & $360 \mathrm{ng} / \mathrm{mL}$ & 42.4 & 93.9 & \\
\hline & & & & CA 19-9 & $11.0 \mathrm{mg} / \mathrm{ml}$ & 30.3 & 91.8 & \\
\hline & \multirow{2}{*}{$\begin{array}{l}\text { Yamamoto et } \\
\text { al. }^{15}(2007)\end{array}$} & \multirow[t]{2}{*}{229} & & CEA & $0.4 \mathrm{ng} / \mathrm{mL}$ & NA & NA & \multirow{2}{*}{$\begin{array}{l}\text { Prediction of peritoneal } \\
\text { recurrence patterns and } \\
\text { prognosis }\end{array}$} \\
\hline & & & & CA 125 & $200 \mathrm{ng} / \mathrm{mL}$ & NA & NA & \\
\hline & \multirow{2}{*}{$\begin{array}{l}\text { Yamamoto et } \\
\text { al. }^{22}(2014)\end{array}$} & \multirow[t]{2}{*}{193} & & CEA & $0.5 \mathrm{ng} / \mathrm{mL}$ & 89.3 & 83.5 & \multirow{2}{*}{$\begin{array}{l}\text { Prediction of peritoneal } \\
\text { dissemination, peritoneal } \\
\text { recurrence patterns, and } \\
\text { prognosis }\end{array}$} \\
\hline & & & & CA $72-4$ & $1.3 \mathrm{ng} / \mathrm{mL}$ & 77.0 & 84.4 & \\
\hline & \multirow{2}{*}{$\begin{array}{l}\text { Mandorwski et } \\
\text { al. }^{14}(2002)\end{array}$} & \multirow[t]{2}{*}{40} & \multirow[t]{2}{*}{24} & CEA & $62 \mathrm{ng} / \mathrm{g}$ protein & 60 & 87 & Most sensitive marker in the PLF \\
\hline & & & & CA 72-4 & $1214 \mathrm{U} / \mathrm{g}$ protein & 55 & 96 & $\begin{array}{l}\text { Most sensitive marker in the } \\
\text { serum }\end{array}$ \\
\hline & Li et al. ${ }^{23}(2005)$ & 64 & 8 & CEA & $100 \mathrm{ng} / \mathrm{g}$ protein & 94.7 & 82.2 & $\begin{array}{l}\text { Prediction of peritoneal } \\
\text { recurrence and prognosis }\end{array}$ \\
\hline & $\begin{array}{l}\text { Fernandes et } \\
\text { al. }^{24}(2007)\end{array}$ & 32 & 18 & CA $72-4$ & $0.61 \mathrm{U} / \mathrm{mL}$ & 40.6 & 100.0 & $\begin{array}{l}\text { Correlation with LN metastasis, } \\
\text { serosal involvement, and more } \\
\text { advanced stage }\end{array}$ \\
\hline & \multirow{2}{*}{$\begin{array}{l}\text { Crepaldi-Filho } \\
\text { et al. }^{12}(2008)\end{array}$} & \multirow[t]{2}{*}{46} & & CEA & $0.2 \mathrm{ng} / \mathrm{mL}$ & 91.0 & 72.0 & \multirow{2}{*}{$\begin{array}{l}\text { Associated with more advanced } \\
\text { stage }\end{array}$} \\
\hline & & & & CA $19-9$ & $2.2 \mathrm{U} / \mathrm{mL}$ & 69.0 & 38.9 & \\
\hline & \multirow[t]{2}{*}{$\begin{array}{l}\text { Hoskovec et } \\
\text { al. }^{25}(2017)\end{array}$} & \multirow[t]{2}{*}{105} & \multirow[t]{2}{*}{12} & CEA & $4.7 \mu \mathrm{g} / \mathrm{L}$ & 53.0 & 100.0 & \multirow[t]{2}{*}{$\begin{array}{l}\text { Prediction of peritoneal } \\
\text { dissemination }\end{array}$} \\
\hline & & & & CA19-9 & $37 \mathrm{U} / \mathrm{mL}$ & & & \\
\hline & $\begin{array}{l}\text { Ito et }_{\text {al. }}^{26} \\
(2005)\end{array}$ & 197 & & $\begin{array}{l}\text { RT-PCR for } \\
\text { CEA mRNA }\end{array}$ & 0.1 & 89.7 & 82.7 & $\begin{array}{l}\text { Prediction of peritoneal } \\
\text { recurrence and prognosis }\end{array}$ \\
\hline & $\begin{array}{l}\text { Wang et al. }{ }^{27} \\
(2005)\end{array}$ & 40 & & & $200 \mathrm{ng} / \mathrm{g}$ protein & 66.7 & 89.3 & $\begin{array}{l}\text { Prediction of peritoneal } \\
\text { dissemination and recurrence }\end{array}$ \\
\hline & $\begin{array}{l}\text { Takata et al. } \\
\text { (2014) }\end{array}$ & 104 & & $\begin{array}{l}\text { RT-PCR for } \\
\text { mRNA of CEA }\end{array}$ & & NA & NA & $\begin{array}{l}\text { Prediction of peritoneal } \\
\text { recurrence and prognosis }\end{array}$ \\
\hline & $\begin{array}{l}\text { Tamura et al. } \\
\text { (2014) }\end{array}$ & 141 & & and CK20 & & NA & NA & $\begin{array}{l}\text { Prediction of peritoneal } \\
\text { recurrence and prognosis }\end{array}$ \\
\hline Ascites & $\begin{array}{l}\text { Jung et al. } \\
\text { (2010) }\end{array}$ & 119 & & CEA & $5.0 \mathrm{ng} / \mathrm{mL}$ & 92.5 & 24 & Prediction of prognosis \\
\hline & Noh et al. ${ }^{30}$ & 93 & & CEA & & 86.7 & & \\
\hline & (2011) & & & MMP-2 & $8.6 \mathrm{ng} / \mathrm{mL}$ & 93.3 & & $\begin{array}{l}\text { Diagnostic and prognostic value } \\
\text { of peritoneal carcinomatosis }\end{array}$ \\
\hline & & & & MMP-9 & $0.14 \mathrm{ng} / \mathrm{mL}$ & 35.2 & & \\
\hline & Yun et al. ${ }^{17}$ & 92 & 73 & miR-181b-5p & & 87.5 & 78.8 & Diagnostic value of peritoneal \\
\hline & (2019) & & & CEA & $2.2 \mathrm{ng} / \mathrm{mL}$ & 87.4 & 81.8 & carcinomatosis \\
\hline & & & & $\begin{array}{l}\text { miR-181b-5p+ } \\
\text { CEA }\end{array}$ & & 82.5 & 96.7 & \\
\hline & Present study & 138 & 64 & CEA & $2.3 \mathrm{ng} / \mathrm{mL}$ & 85.5 & 96.9 & Diagnostic value of peritoneal \\
\hline & & & & CA $19-9$ & $10.2 \mathrm{U} / \mathrm{mL}$ & 79.0 & 100.0 & carcinomatosis \\
\hline & & & & CA $72-4$ & $1.7 \mathrm{U} / \mathrm{mL}$ & 61.6 & 89.0 & \\
\hline & & & & $\mathrm{CEA}+\mathrm{CA} 72-4$ & 5.1 & 86.2 & 100.0 & \\
\hline & & & & CEA+CA 19-9 & 19.9 & 80.4 & 93.8 & \\
\hline & & & & $\begin{array}{l}\text { CEA+CA 72-4 } \\
\text { +CA 19-9 }\end{array}$ & 21.4 & 85.5 & 93.8 & \\
\hline
\end{tabular}

PLF, peritoneal lavage fluid; CEA, carcinoembryonic antigen; CA 125, cancer antigen 125; CA 19-9, carbohydrate antigen 19-9; NA, not available; CA 72-4, cancer antigen 72-4; RT-PCR, reverse transcription polymerase chain reaction; CK-20, cytokeratin-20; MMP-2, matrix metalloproteinase-2; LN, lymph node. 
study is the first to confirm that combined analysis of ascites tumor markers could improve the diagnostic accuracy for GC-ascites in large scale.

In the present study, the levels of CEA, CA 72-4, and CA 19-9 were significantly higher in GC-ascites than in LC-ascites, but the levels of CA 125 were similar. CA 125 is widely used for the diagnosis and surveillance of ovarian cancer. It has also been suggested to be a marker of other gynecologic tumors, including fallopian tube, endometrial, and exocervical cancers, as well as non-gynecologic tumors, including gastrointestinal tract cancers. ${ }^{18}$ However, CA 125 is neither tumor-nor tissue-specific and is elevated in various benign conditions, such as LC, endometriosis, pelvic inflammatory disease, and other disorders associated with ascites or pleural effusion. ${ }^{19,20}$ In this study, CA 125 was increased in both LC-ascites and GC-ascites, which is consistent with the findings of previous studies suggesting that CA 125 is associated with the presence of serosal fluids and the serosal involvement of disease, regardless of the disease origin. ${ }^{19}$

In the diagnosis of malignancy, minimizing the falsepositive rate, which is calculated as "1-specificity," is as important as increasing the sensitivity, because it has a large impact on prognosis and further treatment. The ROC curve analysis of each tumor marker showed that CEA had the highest discriminative power (AUC, 0.952) for the diagnosis of GC-ascites, with $85.5 \%$ sensitivity and 96.8\% specificity. To increase the sensitivity while maintaining the high specificity, we combined CEA with other tumor markers. The sum of CEA and CA 72-4 presented the highest diagnostic power for GC-ascites, with an AUC of $0.966,86.2 \%$ sensitivity, and $100 \%$ specificity. In the subgroup analyses of both cytology positive and negative groups, CEA had similar excellent diagnostic power in both groups, with AUC of 0.939 and 0.982 , respectively. Combination of CEA and CA 72-4 also presented the highest discriminative power for GC-ascites in both groups, with AUC of 0.985 and 0.983 , respectively. Ascites tumor markers, especially combination of CEA and CA 72-4 could assist diagnosing GC-ascites even in the cases of absence of malignant cells in cytologic examination. Compared to the sensitivity of cytologic examination which is only 69\%, combining multiple ascites tumor markers could provide high diagnostic sensitivity and specificity in GC patients. Also, the test procedure is simple and reproducible, it could be easily introduced in clinical practice for the diagnosis of GC related malignant ascites.

The current study has some limitations. First, there were no matched serum samples; therefore, we could not confirm whether the combined analysis of multiple ascites tumor markers is superior to serum tumor markers for the diagnosis of GC-ascites. Second, as we collected anonymized ascites samples from the National Biobank of Korea, which originated from multiple centers, detailed clinical information, including survival, GC treatment history, and response to treatment, could not be obtained. Therefore, we could not confirm the prognostic role of the combination of ascites tumor markers in GC with peritoneal carcinomatosis in this study. Finally, considering the costeffectiveness, measurement of multiple tumor markers could somewhat increase the economic burden during the diagnosis. Compare to the invasive diagnostic procedure like explorative laparotomy, testing the tumor markers in the ascites is relatively simple, non-invasive, and inexpensive diagnostic method for the GC-ascites. Considering the loss of timely treatment opportunity due to delayed diagnosis, measurement of tumor markers in ascites is considered a relatively reasonable method. Despite these limitations, combinational analysis of ascites tumor markers is a simple and strong method for the diagnosis of GC-ascites irrespective of the presence of malignant cells. Timely diagnosis of peritoneal carcinomatosis and prompt decision for the appropriate treatment is crucial for the better prognosis of gastric cancer patients, especially when the ascites is the only presentation of suggesting stage IV disease or recurrence after surgery. Combining the analysis of ascites tumor markers to the cytologic examination could improve the diagnostic sensitivity and could be helpful to apply the proper treatments to the patients. Future studies with serial analyses of ascites tumor markers before and during treatment could provide information on the predictive value of tumor markers in $\mathrm{GC}$ and the potential role of changes in ascites tumor markers as an early biomarker for predicting treatment response.

In conclusion, tumor markers in ascites samples, in- 
cluding CEA, CA 72-4, and CA 19-9, could facilitate to diagnose GC-ascites, make up for the low sensitivity of cytologic method. Combining the measurements of multiple tumor markers could further increase the sensitivity and specificity for the diagnosis of malignant ascites in GC patients.

\section{ACKNOWLEDGEMENT}

This research was supported by a Basic Science Research Program through the National Research Foundation of Korea, funded by the Ministry of Education, Science and Technology (2017R1A5A2015541 and 2019R1A2C1007401). All ascites samples and data in this study were provided by the Biobank of Chungbuk National University Hospital, Gyeongsang National University Hospital, Chonnam National University Hwasun Hospital, and Chonbuk National University Hospital, members of the Korea Biobank Network, which are supported by the Ministry of Health, Welfare and Family Affairs. All ascites samples derived from the Korea Biobank Network were obtained with informed consent under institutional review board-approved protocols. We would like to thank Editage (www.editage.co.kr) for English language editing.

\section{CONFLICT OF INTEREST}

No potential conflict of interest relevant to this article was reported.

\section{ORCID}

\begin{tabular}{|c|c|}
\hline Yaewon Yang & (D) https://orcid.org/0000-0002-1773-134X \\
\hline Hong Jun Kim & (D) https://orcid.org/0000-0002-0411-668X \\
\hline Se-il Go & (D) https://orcid.org/0000-0002-3025-6096 \\
\hline Woo Kyun Bae & ps://orcid.org/0000-0003-0267-1943 \\
\hline Eun-Kee Song & (1) https://orcid.org/0000-0001-8836-0294 \\
\hline Seonggyu Byeon & ://orcid.org/0000-0002-4989-002X \\
\hline Hee Kyung Kim & (D) https://orcid.org/0000-0001-7416-4727 \\
\hline Yusook Jeong & ps://orcid.org/0000-0002-3614-80 \\
\hline hyun Kwon & (1) https://orcid.org/0000-0001-8128-33 \\
\hline i Hyeong Lee & (D) https://orcid.org/0000-0002-7830-5 \\
\hline Bok C & (D) https://orcid.org/0000-0002-9711 \\
\hline
\end{tabular}

Seung-Myoung Son (D) https://orcid.org/0000-0002-1646-4649

Dae Hoon Kim (D) https://orcid.org/0000-0001-9873-4907

Hyo Yung Yun (D) https://orcid.org/0000-0002-5592-1371

Hye Sook Han (D) https://orcid.org/0000-0001-6729-8700

\section{REFERENCES}

1. Sung H, Ferlay J, Siegel RL, et al. Global cancer statistics 2020: GLOBOCAN estimates of incidence and mortality worldwide for 36 cancers in 185 countries. CA Cancer J Clin 2021;71:209-249.

2. Thomassen I, van Gestel YR, van Ramshorst B, et al. Peritoneal carcinomatosis of gastric origin: a population-based study on incidence, survival and risk factors. Int J Cancer 2014;134:622-628.

3. Duffy MJ. Role of tumor markers in patients with solid cancers: a critical review. Eur J Intern Med 2007;18:175-184.

4. Căinap C, Nagy V, Gherman A, et al. Classic tumor markers in gastric cancer. Current standards and limitations. Clujul Med 2015;88:111-115.

5. Emoto S, Ishigami H, Yamashita H, Yamaguchi H, Kaisaki S, Kitayama J. Clinical significance of CA 125 and CA $72-4$ in gastric cancer with peritoneal dissemination. Gastric Cancer 2012; 15:154-161.

6. Liang Y, Wang W, Fang C, et al. Clinical significance and diagnostic value of serum CEA, CA 19-9 and CA 72-4 in patients with gastric cancer. Oncotarget 2016;7:49565-49573.

7. Ahadi M, Tehranian S, Memar B, et al. Diagnostic value of carcinoembryonic antigen in malignancy-related ascites: systematic review and meta-analysis. Acta Gastroenterol Belg 2014;77: 418-424.

8. Kaleta EJ, Tolan NV, Ness KA, O'Kane D, Algeciras-Schimnich A. CEA, AFP and CA 19-9 analysis in peritoneal fluid to differentiate causes of ascites formation. Clin Biochem 2013;46:814-818.

9. Liu F, Kong X, Dou Q, et al. Evaluation of tumor markers for the differential diagnosis of benign and malignant ascites. Ann Hepatol 2014;13:357-363.

10. Zhu FL, Ling AS, Wei Q, Ma J, Lu G. Tumor markers in serum and ascites in the diagnosis of benign and malignant ascites. Asian Pac J Cancer Prev 2015;16:719-722.

11. Yajima K, Kanda T, Ohashi M, et al. Clinical and diagnostic significance of preoperative computed tomography findings of ascites in patients with advanced gastric cancer. Am J Surg 2006;192:185-190.

12. Crepaldi-Filho R, Palma RT, Giusti MF, Bueno Mde A, Silva PS, Waisberg J. Levels of carcinoembryonic antigen and CA 19-9 in the sera and peritoneal washing of patients undergoing surgical treatment for gastric carcinoma. Arq Gastroenterol 2008;45: 219-224.

13. Jung M, Jeung HC, Lee SS, et al. The clinical significance of ascitic fluid CEA in advanced gastric cancer with ascites. J Cancer Res Clin Oncol 2010;136:517-526.

14. Mandorwski S, Lourenço LG, Forones NM. CA 72-4 and CEA in serum and peritoneal washing in gastric cancer. Arq Gastroenterol 
2002;39:17-21.

15. Yamamoto M, Baba H, Toh Y, Okamura T, Maehara Y. Peritoneal lavage CEA/CA 125 is a prognostic factor for gastric cancer patients. J Cancer Res Clin Oncol 2007;133:471-476.

16. Xiao Y, Zhang J, He X, Ji J, Wang G. Diagnostic values of carcinoembryonic antigen in predicting peritoneal recurrence after curative resection of gastric cancer: a meta-analysis. Ir J Med Sci 2014;183:557-564.

17. Yun J, Han SB, Kim HJ, et al. Exosomal miR-181b-5p downregulation in ascites serves as a potential diagnostic biomarker for gastric cancer-associated malignant ascites. J Gastric Cancer 2019;19:301-314.

18. Bergmann JF, Bidart JM, George M, Beaugrand M, Levy VG, Bohuon C. Elevation of CA 125 in patients with benign and malignant ascites. Cancer 1987;59:213-217.

19. Topalak O, Saygili U, Soyturk M, et al. Serum, pleural effusion, and ascites CA-125 levels in ovarian cancer and nonovarian benign and malignant diseases: a comparative study. Gynecol Onco 2002;85:108-113.

20. Zuckerman E, Lanir A, Sabo E, et al. Cancer antigen 125: a sensitive marker of ascites in patients with liver cirrhosis. Am J Gastroenterol 1999;94:1613-1618.

21. Yamamoto M, Baba H, Kakeji Y, et al. Prognostic significance of tumor markers in peritoneal lavage in advanced gastric cancer. Oncology 2004;67:19-26.

22. Yamamoto M, Yoshinaga K, Matsuyama A, Tsutsui S, Ishida T. CEA/CA 72-4 levels in peritoneal lavage fluid are predictive factors in patients with gastric carcinoma. J Cancer Res Clin Oncol 2014;140:607-612.

23. Li JK, Zheng M, Miao CW, Zhang JH, Ding GH, Wu WS.
Peritoneal lavage cytology and carcinoembryonic antigen determination in predicting peritoneal metastasis and prognosis of gastric cancer. World J Gastroenterol 2005;11:7374-7377.

24. Fernandes LL, Martins LC, Nagashima CA, Nagae AC, Waisberg DR, Waisberg J. CA 72-4 antigen levels in serum and peritoneal washing in gastric cancer. Correlation with morphological aspects of neoplasia. Arq Gastroenterol 2007;44:235-239.

25. Hoskovec D, Varga J, Dytrych P, Konecna E, Matek J. Peritoneal lavage examination as a prognostic tool in cases of gastric cancer. Arch Med Sci 2017;13:612-616.

26. Ito S, Nakanishi H, Kodera Y, Mochizuki Y, Tatematsu M, Yamamura Y. Prospective validation of quantitative CEA mRNA detection in peritoneal washes in gastric carcinoma patients. $\mathrm{Br}$ J Cancer 2005;93:986-992.

27. Wang JY, Lin SR, Lu CY, et al. Gastric cancer cell detection in peritoneal lavage: RT-PCR for carcinoembryonic antigen transcripts versus the combined cytology with peritoneal carcinoembryonic antigen levels. Cancer Lett 2005;223:129-135.

28. Takata A, Kurokawa Y, Fujiwara Y, et al. Prognostic value of CEA and CK20 mRNA in the peritoneal lavage fluid of patients undergoing curative surgery for gastric cancer. World J Surg 2014;38:1107-1111.

29. Tamura S, Fujiwara Y, Kimura Y, et al. Prognostic information derived from RT-PCR analysis of peritoneal fluid in gastric cancer patients: results from a prospective multicenter clinical trial. J Surg Oncol 2014;109:75-80.

30. Noh S, Jung JJ, Jung M, et al. MMP-2 as a putative biomarker for carcinomatosis in gastric cancer. Hepatogastroenterology 2011; 58:2015-2019. 